\title{
LoRa Physical Layer Principle and Performance Analysis
}

\author{
Guillaume Ferré, and Audrey Giremus \\ Laboratoire de l'Intégration du Matériau au Système \\ 351 cours de la libération, 33400 Talence, France, \\ Emails:\{guillaume.ferre,audrey.giremus\}@ims-bordeaux.fr
}

\begin{abstract}
The Internet of Things (IoT) finds widespread applications spanning from consumer services such as smart home or domotic to strategic use cases, including infrastructure or energy management. Different technologies have been proposed to support IoT and this paper aims at analyzing the Long Range (LoRA) one. After introducing the main principles of the physical layer, the main contribution is a theoretical performance analysis. Closed-form expression of the symbol and bit error probabilities are derived that are validated by simulation results.
\end{abstract}

\section{INTRODUCTION}

Internet of Things (IoT) has revolutionized ubiquitous computing with multitude of applications. IoT perfectly joins in the future evolution towards the fifth generation of wireless communications technology $(5 \mathrm{G})$. It offers the possibility to connect different objects through the internet so that they can communicate efficiently and reliably, making a house, a car or a city intelligent. Making devices communicate does not represent a big challenge. In general, an object would not have a lot of information to transmit or to receive, and according to use cases, it can communicate sparsely, for instance once a day, a week, a month or even a year. The major difficulty rather stems from the number of objects or even from the battery life of battery-powered devices.

The IoT and Connected Objects $(\mathrm{CO})$ are becoming a reality with the deployment of networks like SigFox [1] or those of Orange and Bouygues Telecoms designed by Semtech, Long Range (LoRa) [2]. Indeed, these technologies offer the possibility to connect any object to the Internet through unlicensed bands. Simultaneously with these low throughput solutions operating in unlicensed bands, 3GPP introduced the normalization of several standards dedicated to the IoT which can use the infrastructure of $2 \mathrm{G}$ and $4 \mathrm{G}$ networks, and thus licensed bands. These standards are: Extended Coverage GSM (EC-GSM), Narrow band IoT (NB-IoT) and LTE - Machine communication type (LTE-M). Since IoT applications are being very numerous, there is not yet a technology able to handle all of them efficiently. Thus, a part of deployed or in progress solutions appears rather as complementary and not competing ones.

This paper focuses on the LoRa technology. It presents first the LoRA physical layer concept and then a performance analysis in terms of error probability. The remainder of the paper is organized as follows. In section II, the LoRa physical layer is introduced. Section III details the approach illustrating bit and symbol error probability expressions related to LoRa modulation. Before concluding our work, simulation results and evaluation are proposed and commented.

\section{LORA PHYSICAL LAYER}

The LoRa technology was developed by a french company called Cycleo, and then acquired and patented by Semtech which is at present selling LoRa chips. The patent [3] provides valuable information on the physical layer, particularly the used modulation scheme which is based on spectrum spreading: Chirp Spread Spectrum (CSS) [4]. Several Spreading Factors (SF) are defined to control the bit rate, improve the range and decrease energy consumption.

LoRa operates in Industrial, Scientific and Medical (ISM) bands over 169, 433, $868 \mathrm{MHz}$ (in Europe) and $915 \mathrm{MHz}$. In order to limit interferences, regulatory authorities specified a duty cycle ranging from 0.1 to $1 \%$ depending on the used sub-band. While other IoT technologies are proprietary, LoRa network management is open and every person has the possibility to deploy LoRa stations or networks and to offer services as long he respects spectrum use regulations. The upper layers to LoRa can be proprietary or standardized. The most popular standard is LoRaWAN, which is implemented by LoRa alliance [5].

\section{A. Physical Layer Principle}

Initially, the binary information flow generated from the MAC layer is divided into subsequences, each of length $S F \in[7 \ldots 12]$. The set of $S F$ consecutive bits constitutes a symbol. The number of possible symbols is hence equal to $M=2^{S F}$. In the LoRa context, $S F$ indicates also the Spreading Factor (SF). Therefore, the relation between the bit rate $D_{b}$ and the symbol rate $D_{s}$ can be written as: $D_{s}=D_{b} / S F$. Spread spectrum is obtained through a signal known as chirp that varies continuously and linearly in frequency. When the derivative of the frequency variation is positive then we deal with an up chirp, conversely it is a down chirp.

The chirps are complex signals, generated in baseband. When the chirp is up or down over the entire symbol period $T_{s}$, which is also called the signaling interval, it is identified as raw chirp. Its complex envelope is mathematically expressed as follows, for $t \in\left[-\frac{T_{s}}{2}, \frac{T_{s}}{2}\right]$ :

$$
s_{l}(t)=e^{j 2 \pi f_{c}(t) t}, \text { with } f_{c}(t)= \pm \frac{B}{T_{s}} t,
$$




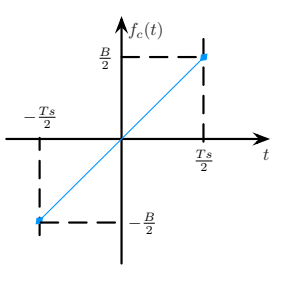

(a)

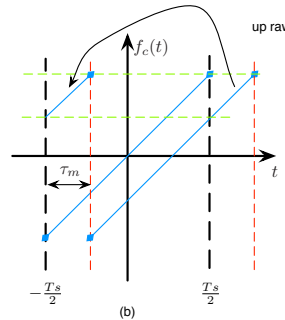

(c)

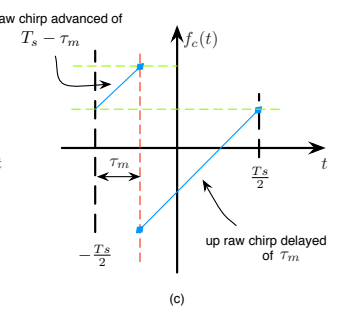

Figure 1. Symbol $\rightarrow$ chirp association process - (a) up raw chirp - (b) process illustration - (c) chirp associated to the $m$ th symbol

where $B$ is the frequency excursion of the transmitted chirp. The '+' and '-' signs stand for up and down raw chirp respectively.

For digital communication systems with no spreading spectrum, the bandwidth used by the transmitted signal is proportional to the symbol rate. The coefficient of proportionality that depends on the shaping filter, which is in general a half Nyquist one. In CSS, the signal bandwidth is fixed by $B$ which has the following relationship with $T_{s}$ :

$$
M=B \times T_{s} .
$$

For a fixed bandwidth, we can deduce that an increase of $S F$ leads to a longer symbol duration.

To distinguish between the $M$ different symbols of the constellation, $M$ orthogonal chirps have to be defined so that each symbol exhibits a specific instantaneous phase trajectory. In the sequel, we denote $S_{k}$ the transmitted symbol at time $k T_{s}$ and $S_{k}=m$ its association to the $m^{\text {th }}$ possible discrete symbol with $m \in\{0, \ldots, M-1\}$. The chirp associated to the $m^{\text {th }}$ symbol is then obtained from the raw chirp by applying a delay $\tau_{m}=\frac{m}{B}$ where we recall that $T_{s}=\frac{M}{B}$. However, to guarantee a good synchronization of the chirps both in time and frequency, LoRa imposes the instantaneous phase $\theta(t)=2 \pi f_{c}(t) t$ to be the same for both the beginning and the end of the chirp. It ensues that $\theta\left(-\frac{T_{s}}{2}\right)=\theta\left(\frac{T_{s}}{2}\right)$. To meet this requirement, the raw chirp outside the interval $\left[-\frac{T_{s}}{2}, \frac{T_{s}}{2}\right]$ is cyclically brought back into $\left[-\frac{T_{s}}{2},-\frac{T_{s}}{2}+\tau_{m}\right]$ as illustrated in Fig.1 (a), (b) and (c). Therefore, the modulated chirp related to the transmission of the symbol $m$ decomposes into two parts:

1) for $t \in\left[-\frac{T_{s}}{2},-\frac{T_{s}}{2}+\tau_{m}\right)$, the ramp of the raw chirp (up or down) advanced in time by $\left(T_{s}-\tau_{m}\right)$,

2) for $t \in\left[-\frac{T_{s}}{2}+\tau_{m}, \frac{T_{s}}{2}\right]$, the ramp of the raw chirp (up or down) delayed in time by $\tau_{m}$.

It follows that the up chirp can for instance be expressed as:

$$
\begin{array}{ll}
f_{c}^{m}(t)=\frac{B}{T_{s}}\left(t-\tau_{m}\right)+B & \text { for } t \in\left[-\frac{T_{s}}{2},-\frac{T_{s}}{2}+\frac{m}{B}\right) \\
f_{c}^{m}(t)=\frac{B}{T_{s}}\left(t-\tau_{m}\right) & \text { for } t \in\left[-\frac{T_{s}}{2}+\frac{m}{B}, \frac{T_{s}}{2}\right]
\end{array}
$$

Eventually, the complex envelope of the transmitted signal is

$$
s(t)=\sum_{k \in \mathbb{Z}} e^{j 2 \pi\left(t-k T_{s}\right) f_{c}^{k}\left(t-k T_{s}\right)}
$$

where $f_{c}^{k}(t)$ corresponds to the modulated chirp related to symbol transmitted at time $k T_{s}$.

\section{B. Symbol Estimation}

In the following, we consider perfect time and frequency synchronizations. As a consequence, the received signal sampled at $T_{e}$ and denoted by $y(n T e)$ can be written as

$$
y(n T e)=\sum_{k \in \mathbb{Z}} e^{j 2 \pi\left(n T e-k T_{s}\right) f_{c}^{k}\left(n T e-k T_{s}\right)}+w(n T e),
$$

where $w(n T e)$ represents the complex noise assumed white, Gaussian and circular.

The transmitted symbols are detected by multiplying every segment with $T_{s}$ period of the complex envelope of the received signal, denoted $y(n T e)$, by the conjugate version of the raw chirp used in the transmitter (up or down). If we consider that the channel did not introduce interferences between chirps, or if a guard interval between chirp was introduced in the transmitter, then the digital demodulation of the $m^{\text {th }}$ transmitted symbol $\left(m T_{s}-\frac{T_{s}}{2} \leq t<m T_{s}+\frac{T_{s}}{2}\right)$ corresponds to the processing of $N=\frac{T_{s}^{2}}{T_{e}}$ samples:

$$
r_{m}\left(n T_{e}\right)=y\left(n T_{e}+m T_{s}\right) e^{-j 2 \pi f_{c}\left(n T_{e}\right) n T_{e}}
$$

with $n \in\left[-\frac{N}{2}, \frac{N}{2}-1\right]$. Thus, in this interval, all the terms of the sum in eq.(3) are null, except the term $k=m$. As a consequence, $\forall m \in\{0, \ldots, M-1\}$ :

$$
y\left(n T_{e}+m T_{s}\right)=e^{j 2 \pi f_{c}^{m}\left(n T_{e}\right) n T_{e}}+w\left(n T_{e}+m T_{s}\right) .
$$

In that respect, by inserting (6) into (5), it follows that

$$
r_{m}\left(n T_{e}\right)=x_{m}\left(n T_{e}\right)+w_{m}\left(n T_{e}\right)
$$

where the useful signal is equal to

$$
x_{m}\left(n T_{e}\right)=\left(e^{j 2 \pi f_{c}^{m}\left(n T_{e}\right) n T_{e}}\right) e^{-j 2 \pi f_{c}\left(n T_{e}\right) n T_{e}}
$$

and the noise term:

$$
w_{m}\left(n T_{e}\right)=w\left(n T_{e}+m T_{s}\right) e^{-j 2 \pi f_{c}\left(n T_{e}\right) n T_{e}}
$$

As well, by multiplying both terms of (8), the arguments will be given by

$$
\begin{array}{r}
\left(-2 \pi \frac{m}{T_{s}} n T_{e}+2 \pi B n T_{e}\right) \text { for } n \in\left[-\frac{N}{2},-\frac{N}{2}+\frac{m}{T_{e} B}\right) \\
\left(-2 \pi \frac{m}{T_{s}} n T_{e}\right) \text { for } n \in\left[-\frac{N}{2}+\frac{m}{T_{e} B}, \frac{N}{2}\right)
\end{array}
$$

In addition, when sampling the signal at a rate of $T_{e}=\frac{1}{B}$, we obtain using eq.(2):

$$
r_{m}\left(n T_{e}\right)=e^{-j 2 \pi \frac{m n}{M}}+w_{m}\left(n T_{e}\right)
$$

It should be noted that this choice of sampling frequency induces $M=N$. Indeed, $r_{m}\left(n T_{e}\right)$ is the sum of a complex exponential with a normalized frequency of $-\frac{m}{N}$ and a Gaussian noise. The optimal estimation of $m$ and thus the detection of the associated symbol can be performed by searching for the maximum of $r_{m}\left(n T_{e}\right)$ periodogram.

Given the proposed solution in the patent EP2449690, the 
discrete Fourier Transform (DFT) at a frequency $\frac{k}{N}$ of $N$ samples from $r_{m}\left(n T_{e}\right)$, denoted by $R_{m}[k], k \in[0, N-1]$, is derived as follows

$$
R_{m}[k]=\frac{1}{\sqrt{N}} \sum_{n=-\frac{N}{2}}^{\frac{N}{2}-1} r_{p}\left(n T_{e}\right) e^{-j 2 \pi \frac{n k}{N}} .
$$

By exploiting the DFT periodicity, $R_{m}[k]$ can be expressed as follows

$$
R_{m}[k]=R_{m}[k-N]=\sqrt{N} \delta(k+m-N)+W_{m}[k],
$$

where $W_{m}[k]$ is the DFT of the noise. The latter is white, Gaussian and with the same variance as $w_{m}\left(n T_{e}\right)$ based on DFT properties. An estimation of $m$ is then given by

$$
\hat{m}=N-\underset{k}{\operatorname{argmax}}\left(\left|R_{m}[k]\right|^{2}\right)
$$

\section{BIT AND SYMBOL ERROR PROBABILITIES}

Based on (12) and (13), we focus in this section on deriving closed-form expressions of the symbol and bit error probabilities before channel decoding. These probabilities are denoted by $P_{s}$ and $P_{b}$, respectively.

First of all, we propose to decompose $P_{s}$ according to the law of total probability. For that purpose, we introduce $M$ hypotheses denoted $\left\{\mathrm{H}_{0}, \ldots, \mathrm{H}_{M-1}\right\} . \mathrm{H}_{m}$ means that the $m^{\text {th }}$ symbol is the one that has actually been transmitted. The symbol error probability then writes:

$$
P_{s}=\sum_{m=0}^{M-1} \mathbb{P}\left[\hat{m} \neq m \mid \mathrm{H}_{m}\right] \mathbb{P}\left[\mathrm{H}_{m}\right]
$$

where $\mathbb{P}\left[\hat{m} \neq m \mid \mathrm{H}_{m}\right]$ is the probability that the estimated symbol is not the transmitted one provided hypothesis $\mathrm{H}_{m}$ is true. In the absence of prior information, all the symbols can be considered equiprobable so that $\mathbb{P}\left[\mathrm{H}_{m}\right]=\frac{1}{M}$. Computing $P_{s}$ therefore amounts to express $\mathbb{P}\left[\hat{m} \neq m \mid \mathrm{H}_{m}\right]$.

It should be noted that false symbol estimations occur when noise realizations are misled for periodogram peaks. To account for the noise effect, we propose to rewrite the probability of interest as follows using the chapman-Kolmogorov formula:

$$
\mathbb{P}\left[\hat{m} \neq m \mid \mathrm{H}_{m}\right]=\int \mathbb{P}_{m} g\left(W_{m}[N-m]\right) \mathrm{d} W_{m}[N-m]
$$

where $\mathbb{P}_{m}=\mathbb{P}\left[\hat{m} \neq m \mid \mathrm{H}_{m}, W_{m}[N-m]\right]$ and $W_{m}[N-m]$ is the noise present at the normalized frequency $\frac{N-m}{N}$ where is located the periodogram peak. As for $g$, it stands for the probability density function of $W_{m}[N-m]$ which is Gaussian. Using the complement rule, the first term of the integrand is given by

$$
\mathbb{P}_{m}=1-\underbrace{\mathbb{P}\left[\hat{m}=m \mid \mathrm{H}_{m}, W_{m}[N-m]\right]}_{\overline{\mathbb{P}}_{m}}
$$

The next step consists in developing $\overline{\mathbb{P}}_{m}$. It should be noted that in the following calculations, all the probabilities are conditional to $W_{m}[N-m]$ and $\mathrm{H}_{m}$ but the latter are omitted for the sake of simplicity.

$$
\begin{aligned}
\overline{\mathbb{P}}_{m} & =\mathbb{P}\left[\forall k \neq N-m,\left|R_{m}[k]\right|^{2}<\left|R_{m}[N-m]\right|^{2}\right], \\
& =\mathbb{P}\left[\forall k \neq N-m,\left|W_{m}[k]\right|^{2}<\left|\sqrt{N}+W_{m}[N-m]\right|^{2}\right] .
\end{aligned}
$$

As the noise samples are Gaussian, $\left|W_{m}[k]\right|^{2}$ is $\chi_{2}$ distributed $\forall k$. Taking advantage of the decorrelation of the noise samples, we then obtain :

$$
\begin{aligned}
\overline{\mathbb{P}}_{m} & =\prod_{\substack{k=0 \\
k \neq N}}^{N-1} \mathbb{P}\left[\left|W_{m}[k]\right|^{2}<\left|\sqrt{N}+W_{m}[N-m]\right|^{2}\right], \\
& =\left(F_{\chi_{2}}\left(\frac{\left|\sqrt{N}+W_{m}[N-m]\right|^{2}}{\sigma^{2}}\right)\right)^{N-1}
\end{aligned}
$$

where $F_{\chi_{2}}$ is the cumulative density function of the $\chi_{2}$ distribution with 2 degrees of freedom and $\sigma^{2}$ is the variance of $w_{m}\left(n T_{e}\right)$ which is the same of the one of $W_{m}[k]$ for each integer $k$.

Finally, eq.(15) cannot be computed analytically. However, it can be interpreted as an expectation with regards to the noise distribution:

$$
\mathbb{P}\left[\hat{m} \neq m \mid \mathrm{H}_{m}\right]=\mathrm{E}_{g}\left[\mathbb{P}_{m}\right] .
$$

As a consequence, we suggest using a Monte Carlo approximation. The approach consists in simulating a large number, i.e. $N_{\mathrm{MC}}$, of realizations of $W_{m}[N-m]$. It should be noted that this step is simple and not computationally expensive since the latter is Gaussian. By denoting these samples $\left\{W^{(i)}\right\}$ for $i=1, \ldots, N_{\mathrm{MC}}$, the law of large numbers yields:

$$
\begin{aligned}
& \mathbb{P}\left[\hat{m} \neq m \mid \mathrm{H}_{m}\right] \\
& \simeq \frac{1}{N_{\mathrm{MC}}} \sum_{i=1}^{N_{\mathrm{MC}}} 1-\left(F_{\chi_{2}}\left(\frac{\left|\sqrt{N}+W^{(i)}\right|^{2}}{\sigma^{2}}\right)\right)^{N-1}
\end{aligned}
$$

In the next step, the symbol error probability is used to infer the bit error probability. It is worth noting that even if the symbol is badly detected, at least one of its bits can be accurately estimated. The probability that a bit is wellestimated while the corresponding symbol is erroneous is equal to $2^{S F-1} /\left(2^{S F}-1\right)$. As a result, the bit error probability $P_{b}$ can be expressed by:

$$
P_{b}=\frac{2^{S F-1}}{2^{S F}-1} P_{s}
$$

\section{Simulation RESUlts AND Discussions}

To validate our understanding of the physical layer, we use an appropriate test bench (see Fig. 2 ) to store several LoRa frames that are being a posteriori decoded by a receiver developed in Matlab. These measures were gathered from COs designed and deployed within our laboratory (see Fig. 3).

A Matlab simulator for LoRa signal transmitter/receiver is implemented later to compute the symbol and the bit error rates for different values of $S F$, with $S F \in[7, \ldots, 12]$. For comparison, the simulated rates are compared with analytical 


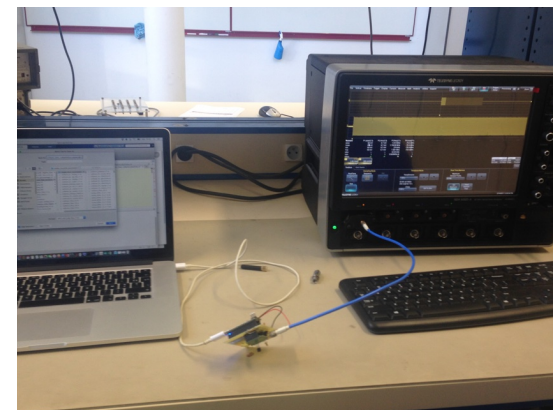

Figure 2. Test bench to obtain real LoRa frames

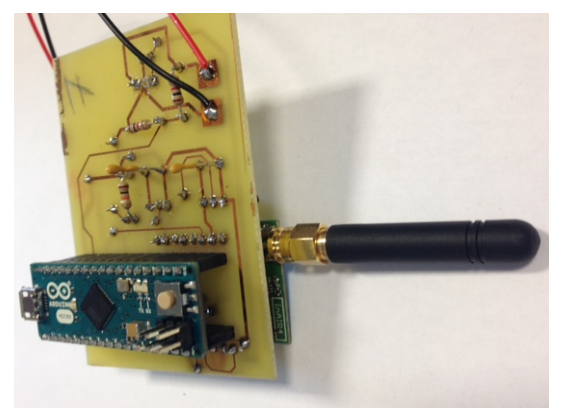

Figure 3. CO used for the measurements

expressions and illustrated in Fig. 4. The error probability values are evaluated as a function to Signal to Noise Ratio (SNR). It is defined as $S N R=\frac{P_{s}}{P_{w}}$ with $P_{s}$ the average power of $s(t)$, obtained integrating its spectral power density and $P_{w}=B N_{0}$ the noise power.

One can notice that the theoretical results are consistent with the obtained simulations since the plots are nearly superimposed. Furthermore, increasing $S F$ by 1 can improve the processing gain by $3 d B$. The results allow one to approve the theoretical adopted approach and to support the gains claimed by the LoRa technology inventor in terms of distance.

Since Sigfox and LoRa are competing technologies, our results will be used to compare their respective bit error

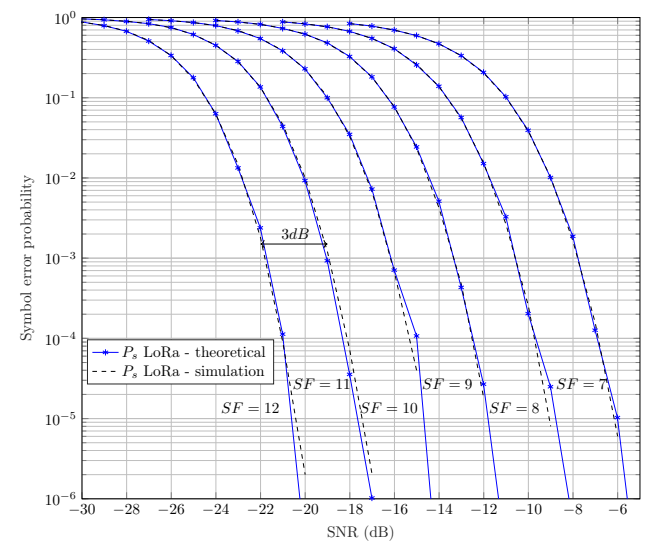

Figure 4. Symbol error probabilities for different values of $S F$.

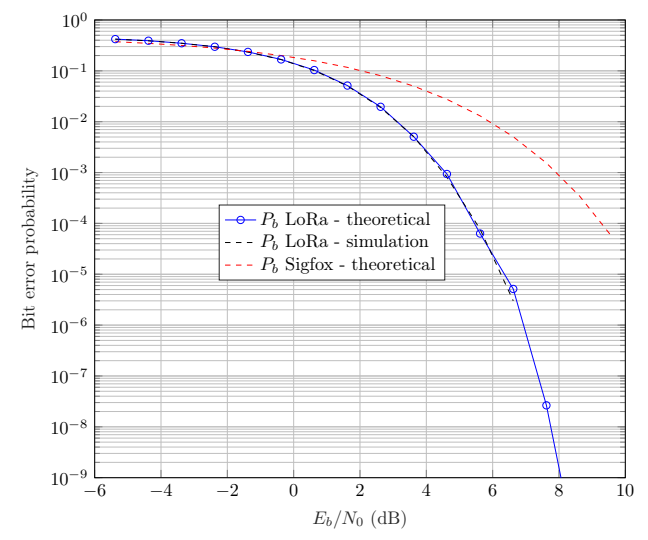

Figure 5. Comparison of bit error probabilities of SigFox and LoRa technologies before channel decoding.

probabilities. Fig. 5 depicts both Sigfox and LoRa bit error probabilities for different values of $\frac{E_{b}}{N_{0}}$, knowing that $\frac{E_{b}}{N_{0}}=$ $\frac{S N R}{\rho}$ where $\rho$ is the spectral efficiency of the communication. In the case of LoRa, we have $\frac{E_{b}}{N_{0}}=S N R \times \frac{2^{S F}}{S F}$.

The digital modulation of Sigfox is the Differential Binary Phase Shift Keying (DBPSK) scheme so that the bit error probability is

$$
P_{b}^{D B P S K}=0.5 \times e^{E_{b} / N_{0}}
$$

We notice that the LoRa technology offers, without channel coding, interesting error rate performance compared to Sigfox. In fact, in case of a service quality equal to $10^{-3}$, LoRa outperforms Sigfox by $3.5 d B$.

\section{CONCLUSION}

In this paper we introduced the LoRa physical layer based on the published patents. In addition, we presented a theoretical evaluation of the symbol and bit error probabilities for this technology. We validated our understanding of the physical layer by decoding a set of LoRa frames that are emitted by Semtech chipsets via a probe station. Then, we plotted the evolution of the symbol and bit error rate that we compared with our theoretical formula. The obtained results validate the formal approach as theory was in accordance with simulation. Finally, given the comparison usually made between Sigfox and LoRa, we stacked on the bit error probability of both technologies as a function of $E_{b} / N_{0}$. As future work, we are analyzing the frequency offset impact on the bit error probability.

\section{REFERENCES}

[1] SigFox, 2016, [accessed on 14.11.2016]. [Online]. Available: http: //www.sigfox.com/en

[2] "Lora modem design guide : Sx1272/3/6/7/8." [Online]. Available: https://www.semtech.com/images/datasheet/LoraDesignGuideSTD.pdf

[3] O. Seller and N. Sornin, "Low power long range transmitter," Aug. 7 2014, uS Patent App. 14/170,170. [Online]. Available: http://www.google.com/patents/US20140219329

[4] A. Berni and W. Gregg, "On the utility of chirp modulation for digital signaling," IEEE Transactions on Communications, vol. 21, no. 6, pp. 748-751, June 1973.

[5] M. Winkler, "Chirp signals for communications," IEEE WESCON Convention Record, p. 7, 1962. 